\title{
How Reliable Are the Properties of Coronal Mass Ejections Measured from a Single Viewpoint?
}

\author{
Laura A. Balmaceda ${ }^{1,5}$ (1) Angelos Vourlidas $^{2,6}$ (10), Guillermo Stenborg ${ }^{3}$ (i), and Alisson Dal Lago ${ }^{4}$ (1) \\ ${ }^{1}$ George Mason University, Fairfax, VA 22030, USA; 1balmace@ gmu.edu \\ ${ }^{2}$ The Johns Hopkins University Applied Physics Laboratory, Laurel, MD 20723, USA \\ ${ }^{3}$ Space Science Division, US Naval Research Laboratory, Washington, DC 20375, USA \\ ${ }^{4}$ National Institute for Space Research (INPE), 12227 São José dos Campos, SP, Brazil \\ Received 2017 December 6; revised 2018 June 25; accepted 2018 June 26; published 2018 August 9
}

\begin{abstract}
We present an analysis of widths and kinematic properties of coronal mass ejections (CMEs) obtained via a supervised image segmentation algorithm, the CORonal SEgmentation Technique (CORSET), on simultaneous observations from the two COR2 telescopes on the Solar Terrestrial Relations Observatory (STEREO) mission, from 2007 May to 2014 September. The sample of 460 events with measurements from two vantage points offers the opportunity to test the accuracy and constraints of single-viewpoint properties that underlie the bulk of CME research to date. In addition, we examine the dependence of the properties on the morphology of the events. The main findings are as follows. (1) The radial speeds derived from different perspectives are in good agreement with a relatively low intrinsic uncertainty of 39\%. (2) Projection effects are more important for determination of CME width rather than for speed. (3) The expansion speeds depend on CME morphology, with loop-type CMEs expanding twice as fast as flux-rope CMEs, possibly underpinning the more explosive nature. (4) Triangulations of CME speed and propagation direction are optimal from viewpoints separated by $60^{\circ}-90^{\circ}$; e.g., between the Lagrangian points $\mathrm{L}_{1}$ and $\mathrm{L}_{5}$ (or $\mathrm{L}_{4}$ ). (5) The projected speeds are underestimated, on average, by at least $20 \%$ when compared to their deprojected (triangulated) values. We also discuss in detail the lessons learned from the application of the CORSET algorithm to event tracking. Our findings should hopefully be a useful guide in the use of (semi)automated algorithms for extraction of CME physical parameters and in the interpretation of singleviewpoint observations (likely to be the norm after the end of the STEREO mission).
\end{abstract}

Key words: catalogs - miscellaneous - Sun: coronal mass ejections (CMEs) - surveys

\section{Introduction}

Simultaneous dual-viewpoint observations of the solar corona have only been available since the launch of the Solar Terrestrial Relations Observatory (STEREO; Kaiser et al. 2008) mission in 2006. As the twin STEREO spacecraft drift around the Sun at $45^{\circ}$ per year relative to each other, they view coronal mass ejection (CME) events from continuously different perspectives. Taking advantage of these capabilities, Vourlidas et al. (2017, hereafter Paper I) compiled a comprehensive list of CMEs using the simultaneous observations from the two COR2 coronagraphs (Howard et al. 2008) on STEREO. The resulting Multi-Viewpoint CME (MVC) catalog is the largest (possibly the only) database to date of events uniquely identified from different perspectives. The MVC catalog database offers a unique resource for investigating projection effects on CME properties.

Until now, key CME measurements for research and modeling of space weather, such as event speed and width, have been derived from a single viewpoint, most recently from observations from the Large Angle and Spectroscopic Coronagraph (Brueckner et al. 1995) on board the Solar and Heliospheric Observatory ( $\mathrm{SOHO}$ ). Since the emission arising from Thomson scattering of the CME-entrained electrons is optically thin, these quantities suffer from projection effects, leading to underestimates of speed and overestimates of CME width (e.g., Burkepile et al. 2004). The long-lived SOHO

\footnotetext{
5 Also at NASA Goddard Space Flight Center, Greenbelt, MD, USA.

${ }^{6}$ Also at IAASARS, National Observatory of Athens, GR-15236, Penteli, Greece.
}

mission resulted in the accumulation of a large body of work on CME properties over almost two solar cycles, including kinematics (Yashiro et al. 2004), energies (Vourlidas et al. 2010), association with solar energetic particles (Gopalswamy et al. 2008), and interplanetary CMEs (Zhang et al. 2007). Because these analyses rely on projected quantities, their statistics and interpretations may be subject to bias, which cannot be reliably assessed without independent observations from a different perspective.

Naturally, there have been several efforts to assess the reliability of single-viewpoint measurements by exploiting the multi-viewpoint observations from STEREO. The majority were focused on deprojection of quantities derived from a single viewpoint via various $3 \mathrm{D}$ reconstruction methodologies (e.g., Temmer et al. 2009; Shen et al. 2013; Lee et al. 2015; Wood et al. 2017). They showed that projection effects can indeed be corrected by means of multi-viewpoint measurements and/or 3D forward modeling. However, these conclusions were based on a relatively small number of events, mainly comprising large and/or halo CME events (because of their obvious importance to space weather). It is difficult to draw robust conclusions on the reliability of single-viewpoint measurements from small samples. In addition, the effects, if any, of CME morphology on the accuracy of these measurements have never been assessed, to our knowledge. For example, one expects that speeds of CMEs with ill-defined fronts (e.g., jets or CMEs lacking coherent structure) may be more uncertain than those of three-part CMEs, or that it may be hard to isolate the true width of a fast CME because of the presence of an enveloping shock. 
Our aim with this paper is to comprehensively assess the robustness of single-viewpoint CME measurements by addressing the small-sample shortcomings of past studies and by investigating the effects of event morphology on the measurements. We mine a unique sample of 460 events with properties measured from two viewpoints as practically unbiased as possible. The events are identified and cross-linked visually in Paper I and their properties (kinematics and geometry) are extracted via a supervised computer vision algorithm called the CORonal SEgmentation Technique (CORSET; Goussies et al. 2010). We believe that a supervised algorithm reduces measurement bias compared to purely manual or purely automated algorithms. It offers the benefits of human-in-theloop for separating the event from the background with automated property extraction. The details are discussed in Section 3.1. Our sample is comprehensive. It covers solar activity from the minimum of cycle 23 to the maximum of cycle 24 , differences in viewing perspective ranging from $0^{\circ}$ to $90^{\circ}$, and all event morphologies we are aware of. It enables us to assess the reliability of past and future single-viewpoint measurements and to understand the effects and limitations of CME morphology on kinematics.

The paper is structured as follows: in Section 2 we describe the methodology for deriving the kinematic and geometric properties of CMEs (Section 2.1), and the deprojected speed and direction of propagation (Section 2.2). The results are presented and discussed in Section 3. First, we summarize the overall performance of the CORSET algorithm in Section 3.1. Then we verify the reliability of the CORSET measurements by comparing the results separately in each telescope in Section 3.2. Only then do we proceed to assess the reliability of singe-viewpoint measurements by comparing the measurements from the two STEREO perspectives against each other in Section 3.3. Finally, we examine the performance of the triangulation approach by analyzing the deprojected speeds and event directions in Section 3.4. In Section 4 we discuss the lessons learned from the use of a supervised detection and measurement technique, and we conclude with a summary of the main results in Section 5.

\section{Methodology}

For the comprehensive study of the projection effects in the determination of the morphological and kinematic properties of CME events, we use the MVC catalog ${ }^{7}$ (Paper I). The catalog is built from the visual inspection of the COR2 images from both the STEREO-A (STA) and STEREO-B (STB) spacecraft for the period 2007-2014. This time period covers the descending phase of cycle 23 and the ascending phase and maximum of cycle 24. The COR2 field of view (FOV) images the solar corona from $\sim 2.5$ to $15 R_{\odot}$, as measured from the Sun's center. One of the novelties of this catalog is the inclusion of a morphology classification for each event and viewpoint. The morphology classification comprises six groups (see Paper I). Three of them have very distinctive characteristics, namely groups F (flux rope), L (loop), and $\mathrm{J}$ (jet); while groups $\mathrm{O}$ (other) and $\mathrm{U}$ (unknown) exhibit an ambiguous appearance, possibly due to overlapping with other structures and/or orientation. The remaining category, group W (wave-like), corresponds to CME-like events that fade away as they travel

\footnotetext{
7 The catalog is available online at https://solar.jhuapl.edu.
}

across the field of view of the coronagraph and do not appear to escape into the heliosphere.

The other novelty of the MVC catalog is the application of the supervised computer vision algorithm, CORSET, to extract the kinematic/geometric properties of the CME. We explain the motivation behind this approach in detail in Paper I and briefly repeat it in the Introduction. We apply the CORSET algorithm to all events in our list irrespective of morphological type, size, apparent direction, or spacecraft location. In this way, we can better evaluate its performance. We use COR2 images corrected for bias and exposure time and divided by the monthly minimum, binned to a size of 1024 by 1024 pixels, which has been proved to not affect the segmentation procedure (Goussies et al. 2010; Braga et al. 2013).

\subsection{Supervised CME Measurements: a Brief Description of the CORSET Algorithm}

From the list of STA and STB COR2 images for each event, we select a frame containing a significant (and clearly distinguishable from the background) portion of the CME to allow the CORSET algorithm to evaluate the textural features of both the foreground (i.e., the CME feature) and the background. This is done by manually tracing, only on this image, a subregion within the outer boundary of the CME and a similarly sized region of the background. This is the supervised part of the technique. The image with the manually selected regions is then passed into the CORSET algorithm, which automatically segments each image in the full time sequence where the event was seen to occur. The time sequence of images is defined in the MVC catalog in Paper I.

Briefly, the CME segmentation (automated part) is based on the analysis of the textural characteristics of the regions initially defined as foreground and background. The textural properties are evaluated via the gray-level co-occurrence matrix (GLCM, Haralick et al. 1973) in both the CME and background subregions separately. The elements of the GLCM are defined as the relative frequencies of occurrence of pairs of gray-level values of adjacent pixels (i.e., separated by a distance of one pixel) in the diagonal direction (see, e.g., Braga et al. 2013, and references therein).

The CORSET algorithm modifies, on the fly, the image representation on which it works. As it proceeds with the second frame and subsequent ones, the algorithm combines a running-difference scheme outside the boundaries of the CME defined in the previous frame, and a base-difference inside the $\mathrm{CME}$ region (referred to as $R_{j-1}$ ). The runningdifference choice accounts for the highly varying conditions of the background corona during the evolution of the CME. On the other hand, a base-difference image is more suitable for evaluating the textural characteristics within the CME region. Thus, the resulting working image $I_{j}^{*}$ in the sequence is obtained in the following manner:

$$
I_{j}^{*}(x)= \begin{cases}I_{j}(x)-I_{\text {base }}(x), & \text { with } x \in R_{j-1} \\ I_{j}(x)-I_{j-n}(x), & \text { with } x \notin R_{j-1}\end{cases}
$$

The base image $I_{\text {base }}$ is a pre-event image. The runningdifference images, on the other hand, result from subtracting the $(j-n)$ th image from the $j$ th image. The lag, $n$, between images is user-defined as an input parameter. By default, $n$ is set to 1 . However, a higher value of $n$ is preferable for slow 

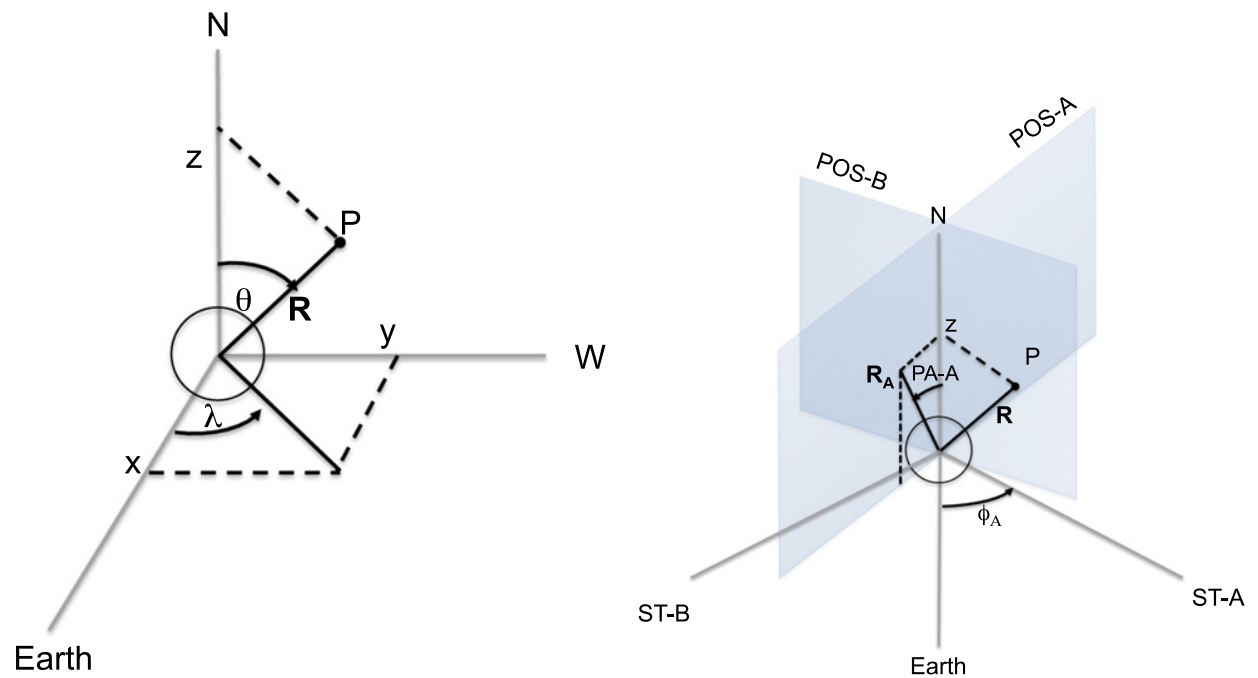

Figure 1. Left: coordinate system. The Sun is located at the center. The point $\mathrm{P}$ represents an arbitrary point in the CME outer boundary at a distance $R$ from the center. Right: sketch showing the positions of STA and $S T B$ spacecraft relative to Earth. $\phi_{A}$ is the separation angle of STA with respect to the Earth. The plane of sky (POS), i.e., the plane where the CME feature is projected, is shown for both spacecraft. For simplicity, only the projected distance $R_{A}$ and its corresponding position angle $\left(\mathrm{PA}_{A}\right)$ for STA are shown.

events (if the detection fails for a given value of $n$, the user can make a new run with a new value-another advantage of the supervised approach).

At each subsequent timestamp, the CME region segmented in the previous frame is expanded isotropically by a small percentage. The GLCM is re-evaluated in order to account for the textural variability from one image to the next. The degree of expansion with respect to the segmented feature in a previous frame is the parameter we call $q$, which is controlled by the user (also as an initial input parameter). In particular, null values of $q$ (i.e., $q=0$ ) imply that no or little expansion is carried out, $q=[4-8]$ are typical values that work for most cases, and $q=16$ is generally used when a shock is detected. Another user-controlled parameter is the size of the angular sector containing the CME event, which is particularly useful for overlapping events. The default value is set to $360^{\circ}$, i.e., the full field of view.

The detection procedure is automatically repeated throughout the sequence of images, which allows the tracking of the event. Morphological operations (such as dilation and erosion) are applied at the end of each frame segmentation analysis to remove the small-scale spatial fluctuations in the resulting CME contour. For full details on the mathematical procedure and formalism, the reader is referred to Goussies et al. (2010).

The automatic calculation of a set of kinematic (radial and expansion speeds) and morphological (angular size and central position angle) CME properties takes place once the CORSET-based segmentation of each frame in the time sequence is finalized. This stage is described in detail in Section 2.3 of Paper I. Note that since the CORSET algorithm identifies the full boundary contour between the CME and the background, height-time measurements for all points along the contour are available (not just at a single point, which is the usual case with manually derived CME measurements), hence allowing for a more comprehensive kinematic analysis of the events.

\subsection{Derivation of the CME Propagation Direction and Speed in $3 D$ Space}

To estimate the direction of propagation and deprojected (true) speeds from the plane-of-sky (POS) (i.e., projected) speeds we use the following triangulation-based technique.

Similarly to Mierla et al. (2008), we use affine geometry for the $3 \mathrm{D}$ reconstruction. In this approach, the CME is assumed to be orthogonally projected onto the POS. This approximation is valid because the Sun-observer distance is sufficiently large compared to the dimensions of the object of interest. In practice, the error introduced is of the order of the CME height over the Sun-observer distance, varying from about 3\% to 6\% in the case of the COR2 coronagraphs.

In this reconstruction, the $3 \mathrm{D}$ position of a given point is described in spherical coordinates $(R, \theta, \lambda)$. As seen in the left panel of Figure 1, the Sun is located at the center. The Earth lies on the $x$ axis. The $z$ axis is perpendicular to the plane of the ecliptic, pointing to north, $\mathrm{N}$. A point $\mathrm{P}$ is arbitrarily located at a distance $R$ away from the Sun, representing a point at the CME boundary. The symbols $\theta$ and $\lambda$ represent the colatitude and the longitude of the vector Sun-P relative to the Earth-Sun line, respectively. The right panel of Figure 1 shows a diagram with the positions of the STEREO spacecraft relative to Earth and their respective planes of sky, POS-A and POS-B. For clarity, we show only the measurements projected onto POS-A. The projected distance $R_{A}$ corresponds to the position angle $\mathrm{PA}_{A}$ measured counterclockwise from the North Pole. STA is located at an angular distance from Earth, $\phi_{A}$, and $S T B$ at $\phi_{B}$. To simplify calculations, we ignore the distance of both spacecraft from the ecliptic, and consider their orbits to be coplanar on the ecliptic plane as done by Mierla et al. (2008). By decomposing each of the measured vectors $\left(R_{A}, R_{B}\right)$ into the orthogonal projections to the $z$ axis and $x y$ plane, and subsequently into the $x$ and $y$ axes, we can write a system of two equations from which the $x$ and $y$ components of the position vector $\boldsymbol{R}$ can be obtained:

$$
\boldsymbol{R}=x \hat{\boldsymbol{\imath}}+y \hat{\boldsymbol{\jmath}}+z \hat{\boldsymbol{k}}
$$



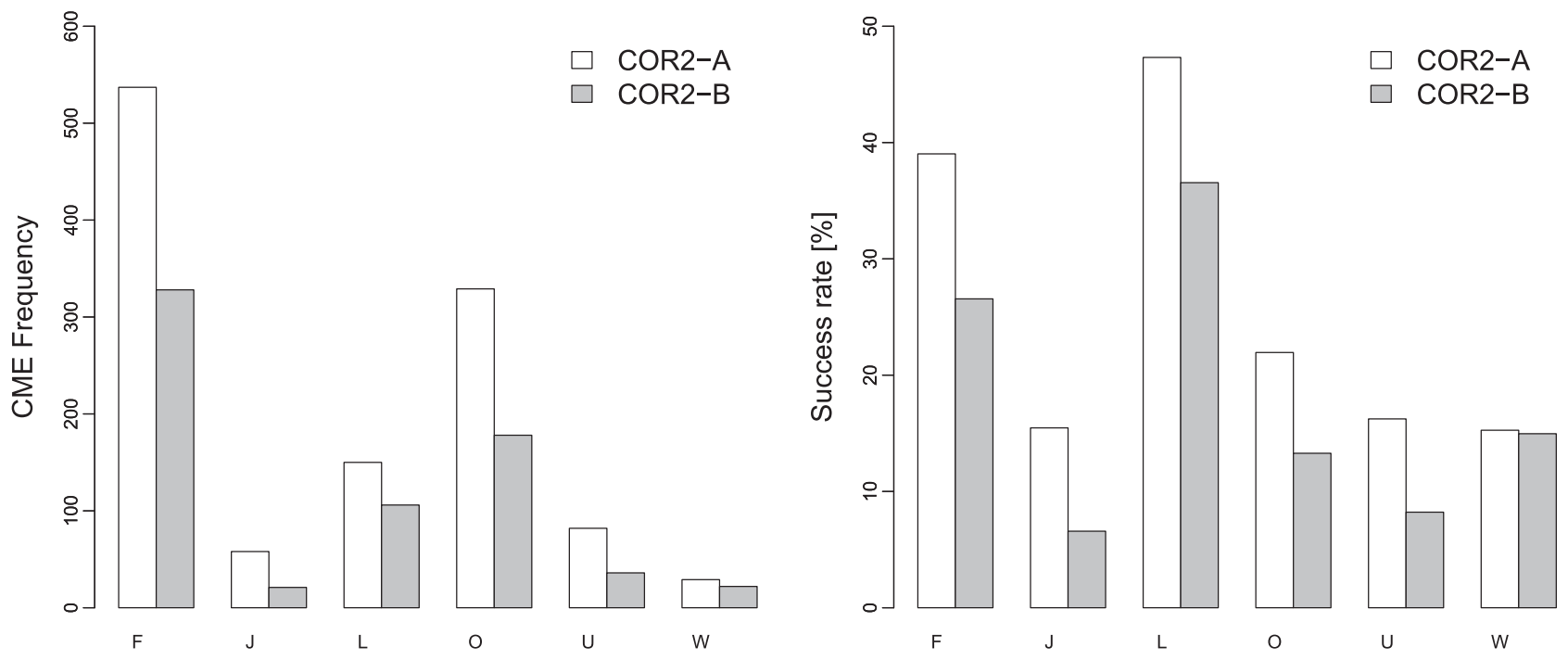

Figure 2. Left: total number of CMEs successfully segmented and tracked by CORSET. Right: success rate of CORSET per morphological type and spacecraft. Different colors correspond to STA and STB respectively.

$$
\begin{gathered}
x=\frac{R_{A} \sin \left(\mathrm{PA}_{A}\right) \cos \left(\phi_{B}\right)-R_{B} \sin \left(\mathrm{PA}_{B}\right) \cos \left(\phi_{A}\right)}{\sin \left(\phi_{A}\right) \cos \left(\phi_{B}\right)+\cos \left(\phi_{A}\right) \sin \left(\phi_{B}\right)} \\
y=-\frac{R_{A} \sin \left(\mathrm{PA}_{A}\right) \sin \left(\phi_{B}\right)+R_{B} \sin \left(\mathrm{PA}_{B}\right) \sin \left(\phi_{A}\right)}{\sin \left(\phi_{A}\right) \cos \left(\phi_{B}\right)+\cos \left(\phi_{A}\right) \sin \left(\phi_{B}\right)} \\
z=R_{A} \cos \left(\mathrm{PA}_{A}\right) \simeq R_{B} \cos \left(\mathrm{PA}_{B}\right) \\
\tan (\lambda)=\frac{y}{x} \\
\tan (\theta)=\frac{\sqrt{x^{2}+y^{2}}}{z} .
\end{gathered}
$$

Note that the $z$ component should be the same for both spacecraft. This can only be attained if both spacecraft follow exactly the same point. In practice, we take $z$ as the average between the two measurements.

By differentiating Equations (2)-(5) with respect to time and assuming that the CMEs propagate radially (i.e., at a constant position angle), we can obtain the components of the true (deprojected) speed of the CME from the measured projected quantities $V_{A}, V_{B}, \mathrm{PA}_{A}$, and $\mathrm{PA}_{B}$,

$$
\begin{gathered}
\boldsymbol{V}=\frac{d x}{d t} \hat{\boldsymbol{\imath}}+\frac{d y}{d t} \hat{\boldsymbol{\jmath}}+\frac{d z}{d t} \hat{\boldsymbol{k}} \\
\frac{d x}{d t}=\frac{V_{A} \sin \left(\mathrm{PA}_{A}\right) \cos \left(\phi_{B}\right)-V_{B} \sin \left(\mathrm{PA}_{B}\right) \cos \left(\phi_{A}\right)}{\sin \left(\phi_{A}\right) \cos \left(\phi_{B}\right)+\cos \left(\phi_{A}\right) \sin \left(\phi_{B}\right)} \\
\frac{d y}{d t}=-\frac{V_{A} \sin \left(\mathrm{PA}_{A}\right) \sin \left(\phi_{B}\right)+V_{B} \sin \left(\mathrm{PA}_{B}\right) \sin \left(\phi_{A}\right)}{\sin \left(\phi_{A}\right) \cos \left(\phi_{B}\right)+\cos \left(\phi_{A}\right) \sin \left(\phi_{B}\right)} \\
\frac{d z}{d t}=V_{A} \cos \left(\mathrm{PA}_{A}\right) \simeq V_{B} \cos \left(\mathrm{PA}_{B}\right) .
\end{gathered}
$$

In computing the deprojected quantities we use the speeds in the fastest direction, the maximum propagation angle (MPA), for both spacecraft. We further assume that the direction of the CME remains constant, i.e., we use a single value each for $\mathrm{PA}_{A}$ and $\mathrm{PA}_{B}$.

\section{Results and Discussion}

\subsection{Application of the CORSET Algorithm to the MVC Catalog}

The CORSET algorithm successfully segmented and tracked 1185 out of 4262 COR2-A events (28\%) and 691 out of 3769 COR2-B events (18\%). For each event, we manually selected the CME and background regions (in only one frame). We defined the following tracking parameters: initial and final frames, base image, lag $n$ between the images for running differences, the circular sector encompassing the CME (only if necessary), and the segmentation parameter: expansion factor $q$. For the interested reader, the set of segmentation and tracking parameters used for each successful event can be found as an ASCII file in the online catalog (http://solar. jhuapl.edu/Data-Products/COR-CME-Catalog.php) in the "date" column.

The selection of an appropriate pre-event image is critical for the success of the segmentation algorithm. When possible, the image closest in time to the first CME appearance was chosen as the base image. However, especially at times of high activity, other coronal features such as streamers or CMEs leave imprints along the CME location. The median time difference between the pre-event and first CME image was $2 \mathrm{hr}$ for the whole set of events. This time difference was less than $5.5 \mathrm{hr}$ for $75 \%$ of the events.

The lag $n$ between images for the running-difference sequences was set to 1 for the majority of the successfully detected events (85\%). The remaining $15 \%$ correspond to slow events, i.e., streamer blowouts, where the front moves little from one frame to the next and hence a larger time separation between images (i.e., $n>1$ ) was necessary.

The expansion factor was set to 8 for most cases. Only $10 \%$ of the events required a different value. We found that this setting did not significantly affect the effectiveness of the segmentation procedure.

We show in Figure 2 the total number of CORSET events per morphological type and spacecraft (left plot) and the corresponding success rate, i.e., the number of CORSETmeasured events over the total number in the MVC catalog (right plot). Most of the successfully segmented events belong to F- and O-type CMEs. When considering the success rate for 

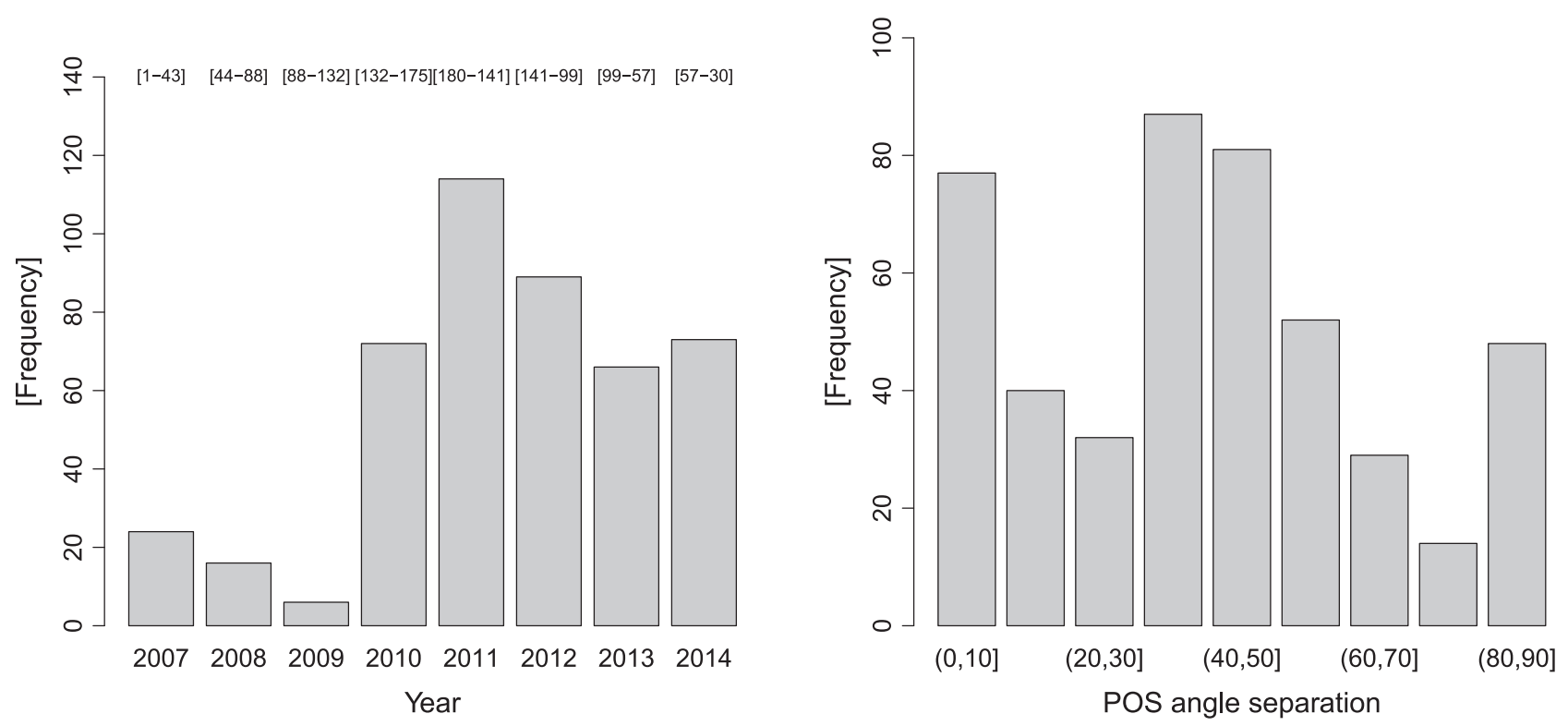

Figure 3. Left: yearly number of unique CMEs detected with CORSET simultaneously in STA and STB images. The range of POS separation angle for each year is indicated on the top. Right: number of CMEs per $10^{\circ}$ bin of POS separation angle.

each type, the best rates are obtained for F- and L-type CMEs, which is unsurprising since these events are usually bright and large in the coronagraph FOV. Almost half of these events were segmented and tracked effectively. The trend is similar for both spacecraft although the success rate of COR2-B is lower than that of COR2-A. This is most probably due to the lower signal-to-noise ratio in COR2-B images compared to COR2-A (Frazin et al. 2012; Vourlidas et al. 2017). Overall, the success rate of CORSET is $\sim 18 \%$ for COR2-B and $\sim 28 \%$ for COR2A. The combined success rate (i.e., the percentage of unique events for which we have measurements from at least one spacecraft) is $\sim 33 \%$. But here we focus on the events measured simultaneously in both spacecraft.

Of the 3558 events simultaneously detected in COR2-A and -B, CORSET segmented and tracked 460 events $(\sim 13 \%)$ covering the full range of spacecraft separation angles. In Figure 3 (left) we show the yearly number of CORSET events and the number of events per $10^{\circ}$ separation angle in the spacecraft POS (right). Naturally, the highest number of events in our sample occurs in 2010-2012, near solar maximum. During this period, the STEREO spacecraft separated from $\sim 130^{\circ}$ to $180^{\circ}$. As the spacecraft approach opposition, their viewing geometry of CMEs gradually becomes similar, reducing any differences due to projection. Opposition gives us the opportunity to validate the CORSET measurements (see Section 3.3). The number of CORSET-tracked events in both spacecraft is quite low in 2009 for two possible reasons: (1) 2009 is very close to the minimum of the cycle. CMEs at solar minimum are intrinsically faint compared to the background corona, making it hard to achieve a good segmentation (the faintness of an event is also an issue with other algorithms employing different mathematical properties, based on the direct experience of one of the authors, A.V., during the adaptation of CACTUs ${ }^{8}$ for the COR2 images); and (2) STA and $S T B$ were in or close to quadrature during this year, and hence events propagating near the POS of one spacecraft will appear as halos in the other, making them hard to track down in

Robbrecht \& Berghmans (2004).
Table 1

Distribution of CMEs According to Morphological Types as Seen by Both Spacecraft and POS Separation Angles

\begin{tabular}{lrrrrr}
\hline \hline \multirow{2}{*}{ POS } & \multicolumn{3}{c}{ Morphological Type } & \multirow{2}{*}{ Total } \\
\cline { 3 - 5 } & & F-F & O-O & F-Other & \\
\hline CORSET & all angles & $58 \%$ & $31 \%$ & $11 \%$ & 460 \\
& $0^{\circ}-30^{\circ}$ & $70 \%$ & $29 \%$ & $1 \%$ & 149 \\
& $30^{\circ}-60^{\circ}$ & $49 \%$ & $35 \%$ & $16 \%$ & 220 \\
& $60^{\circ}-90^{\circ}$ & $64 \%$ & $22 \%$ & $14 \%$ & 91 \\
\hline Catalog & all angles & $34 \%$ & $54 \%$ & $13 \%$ & 3558 \\
& $0^{\circ}-30^{\circ}$ & $36 \%$ & $61 \%$ & $3 \%$ & 947 \\
& $30^{\circ}-60^{\circ}$ & $31 \%$ & $54 \%$ & $15 \%$ & 1602 \\
& $60^{\circ}-90^{\circ}$ & $36 \%$ & $46 \%$ & $18 \%$ & 1009 \\
\hline
\end{tabular}

Note. Top: CORSET events. Bottom: all events in the MVC catalog.

one of the telescopes. The latter indeed greatly reduces the number of simultaneous detections. In contrast to 2009, the success rate in 2007 is higher despite similar conditions at solar minimum. We believe that the difference is due to the small spacecraft separation. The separation angle increased from about only $6^{\circ}$ to $44^{\circ}$ and therefore the differences due to projection effects were less pronounced. This provides support to our second assertion above.

The event morphology plays a role in the success rate of the algorithm, as suggested by Figure 2, which shows the performance for each spacecraft. Because we are using a subsample of our overall CME catalog, we check for any biases arising from this selection effect. We concentrate on what we consider the "real" CMEs: F-, L-, and O-type CMEs, i.e., morphologies consistent with a magnetic flux rope. In Table 1, we summarize the proportions of CMEs in both telescopes for each morphological group, for our sample and the full catalog sample. We include F- and L-CMEs together because we interpreted them as different projections of a flux rope. We break down the event percentages for three bins of POS separation angle. 
The first thing to notice is that most CMEs in our sample correspond to F-F CMEs, while in the full catalog the majority belong to the $\mathrm{O}-\mathrm{O}$ category. This suggests that CMEs in group $\mathrm{O}$ show more diffuse boundaries than F-CMEs. The proportions in the F-Other group, however, are very similar in both samples. When we examine the proportions in each bin of POS separation angle we find an interesting result. A very small percentage (less than 3\%) of F-Other CMEs is found when the separation angle between the spacecraft is less than $30^{\circ}$. This percentage increases for larger separation angles. In the bin $30^{\circ}-60^{\circ}$, the fraction of CMEs seen as flux ropes by one of the spacecraft increases by $12 \%$ (5\% decrease in the $\mathrm{F}-\mathrm{F}$ group and $7 \%$ in the $\mathrm{O}-\mathrm{O}$ group). In the bin $60^{\circ}-90^{\circ}$, it is the fraction of $\mathrm{O}-\mathrm{O}$ CMEs that decreases even more. A similar trend is observed in both samples. We interpret these results as follows. When the views are similar, the morphological type coincides for both spacecraft, even if there are no clear signatures of a flux rope. For a very small percentage, the appearance from one viewpoint is not clear and therefore the event falls in a different category than F-type, most probably due to a very particular orientation of the CME. But this changes as the spacecraft reach larger separations: more CMEs can have a very different appearance from another viewpoint with clear flux-rope signatures in only one perspective. These results support the hypothesis that a larger number of CMEs than reported from single-viewpoint observations correspond to the flux-rope type.

Overall, the discussion above demonstrates that our sample of CORSET-measured CMEs is representative of the solar cycle activity and all possible geometric configurations between the COR2 telescopes, and is mostly filled with "real" CMEs. We therefore expect that the results of our analysis will be applicable to the overall CME database.

\subsection{How Reliable are the CORSET Measurements?}

In Paper I (Section 3.2) we presented the top-level results for the kinematics and morphology of the CME events analyzed with CORSET and compared the yearly values with other available catalogs. Here we expand upon that work to investigate the consistency of CORSET when applied to different instruments (COR2-A and -B, in this case). In addition, we examine (1) whether the CORSET measurements depend on the event morphology and (2) whether the statistics support our implicit connection between morphology and the physical nature of an event.

In Table 2 we summarize the statistics for the radial and expansion speeds and angular width per morphological type and per telescope. We provide the median and its corresponding 95\% confidence interval (in brackets). We calculate speeds using both a linear fit (constant speed) and a quadratic fit (constant acceleration). The radial speeds are computed from the height-time measurements at the central position angle (CPA). Measurements along the leading edge, and the MPA, are also available but are not shown here. There are no significant differences in the average values of linear or quadratic speeds (estimated at $10 R_{\odot}$ ) for all types except for wave-like (group W) CMEs, whose fronts fade away as they cross the FOV. F-CMEs exhibit the smallest range of acceleration on average $\left(1-3 \mathrm{~m} \mathrm{~s}^{-2}\right)$ while L-CMEs exhibit the largest range of decelerations $\left(-45\right.$ to $\left.2 \mathrm{~m} \mathrm{~s}^{-2}\right)$, particularly for COR2-A. Generally, all morphological types except F-CMEs show deceleration in the COR2 FOVs.
The expansion speed is defined simply as the rate of change of the linear distance between the CME flanks at the height of maximum separation in the direction perpendicular to the propagation. The reader is referred to Braga et al. (2013) for details of the procedure employed. Briefly, the expansion speed is calculated as follows. For each frame, we first determine the maximum distance from the solar center. We then measure the linear separation between the outermost points at different distances from the occulter edge border to that maximum height. We use the maximum separation in each frame as the value describing the expansion at a given time. The expansion speed is finally estimated by fitting either a linear or a quadratic equation to the points.

The expansion speeds reported in Table 2 are relative to the central direction of propagation (i.e., the rate of change of the lateral expansion divided by two). Flux-rope CMEs (F-CMEs) show typical expansion speeds of about $120-147 \mathrm{~km} \mathrm{~s}^{-1}$ while loop CMEs (L-CMEs) have the largest speeds, $365-390 \mathrm{~km} \mathrm{~s}^{-1}$. This is consistent with the interpretation proposed by Vourlidas et al. $(2013,2017)$ that the L-CMEs are just flux-rope CMEs with their major axis along the POS (and suggested earlier by Cremades \& Bothmer 2004). If this interpretation is correct, these speeds imply that the CME-entrained flux ropes expand faster along their major axis than along their minor axis, a prediction that should be tested against theory. O-CMEs expand with about the same speeds as F-CMEs, which lends some support to their interpretation as "masked" F-CMEs (Vourlidas et al. 2017). We also see that the jet-like CMEs have similar speeds (and are slower than F-CMEs) from both viewpoints, which is consistent with them being a self-similar expanding narrow structure rather than the result of a projection of a wider object.

The CORSET measurements also provide the angular width of the CME for each image in a given CME time series. To uniquely characterize the CME width, we use two measures: the mean width in the time sequence and the width at $10 R_{\odot}$. The medians of these two parameters are summarized for each CME group in the rightmost panel of Table 2. F-CMEs exhibit typical widths of $\sim 50^{\circ}$, in agreement with past results on the average CME width (e.g., Yashiro et al. 2004; Vourlidas et al. 2010). Again, the almost $2 \times$ larger average width of L-CMEs $\left(90^{\circ}\right)$ compared to F-CMEs suggests that these are F-CMEs seen face-on. The J-CMEs have the smallest widths, typically $\sim 13^{\circ}$, in agreement with their interpretation as jets. O-type CMEs are slightly narrower than F-CMEs. We also report in Table 2 the typical standard deviation within a set of measurements. All CME groups show similar width variations, with the L-CMEs exhibiting the highest standard deviation in the widths.

In Figure 4 we show the distribution of the median CPA and MPA values for $S T A$ (left) and STB (right). We find that $\sim 55 \%$ of the events propagate near the equator, with median CPA values smaller than $30^{\circ}$; about $30 \%$ of the events appear at midCPA values $\left(30^{\circ}-60^{\circ}\right)$ and the remaining $15 \%$ of the events appear at higher CPA values $\left(>60^{\circ}\right)$. Similar results are found for the median MPAs. We do not find significant differences between the CPA and MPA statistics (Figure 4). The residuals of |CPA-MPA| lie below $10^{\circ}$ for at least $80 \%$ of the events, $15 \%$ of the events have residuals between $10^{\circ}$ and $20^{\circ}$, and residuals larger than $20^{\circ}$ exist for only $5 \%$ of events for both spacecraft. 
Table 2

Median Values of Radial Speed, Expansion Speed, and Angular Width of the CMEs as a Function of Morphology and Viewpoint

\begin{tabular}{|c|c|c|c|c|c|c|c|c|c|c|}
\hline \multirow{2}{*}{ Type } & \multirow{2}{*}{ Spacecraft } & \multicolumn{3}{|c|}{ Radial Speed } & \multicolumn{3}{|c|}{ Expansion Speed } & \multicolumn{3}{|c|}{ Angular Width } \\
\hline & & $\begin{array}{c}\text { Linear Fit } \\
\left(\mathrm{km} \mathrm{s}^{-1}\right)\end{array}$ & $\begin{array}{l}\text { Quad. Fit } \\
\left(\mathrm{km} \mathrm{s}^{-1}\right)\end{array}$ & $\begin{array}{l}\text { Accel. } \\
\left(\mathrm{m} \mathrm{s}^{-2}\right)\end{array}$ & $\begin{array}{c}\text { Linear Fit } \\
\left(\mathrm{km} \mathrm{s}^{-1}\right)\end{array}$ & $\begin{array}{l}\text { Quad. Fit } \\
\left(\mathrm{km} \mathrm{s}^{-1}\right)\end{array}$ & $\begin{array}{l}\text { Accel. } \\
\left(\mathrm{m} \mathrm{s}^{-2}\right)\end{array}$ & $\begin{array}{l}\text { At } 10 R_{\odot} \\
\quad(\mathrm{deg})\end{array}$ & $\begin{array}{l}\text { Mean } \\
(\mathrm{deg})\end{array}$ & $\begin{array}{l}\text { Std. Dev. }{ }^{a} \\
\text { (deg) }\end{array}$ \\
\hline \multirow[t]{2}{*}{$\mathrm{F}$} & $\mathrm{A}$ & $330[311,356]$ & $370[344,409]$ & $2[1,3]$ & $120[109,136]$ & $128[116,144]$ & $0[0,1]$ & $53[51,55]$ & $52[49,54]$ & $8[8,9]$ \\
\hline & $\mathrm{B}$ & $388[352,435]$ & $407[384,443]$ & $4[2,6]$ & $147[128,168]$ & $143[125,163]$ & $0[-1,1]$ & $48[46,50]$ & $46[44,49]$ & $8[7,9]$ \\
\hline \multirow[t]{2}{*}{$\mathrm{J}$} & A & $266[189,376]$ & $176[88,344]$ & $-21[-35,-14]$ & $30[22,41]$ & $19[11,32]$ & $-1[-2,0]$ & $13[11,14]$ & $13[11,14]$ & $3[2,4]$ \\
\hline & $\mathrm{B}$ & $384[204,540]$ & $351[178,555]$ & $-16[-45,2]$ & $43[10,54]$ & $54[19,92]$ & $1[-2,6]$ & $13[9,15]$ & $12[10,15]$ & $2[2,4]$ \\
\hline \multirow[t]{2}{*}{$\mathrm{L}$} & A & $621[570,692]$ & $616[550,730]$ & $-5[-12,0]$ & $365[296,405]$ & $338[274,382]$ & $-13[-22,-8]$ & $90[82,94]$ & $87[78,91]$ & $16[15,19]$ \\
\hline & $\mathrm{B}$ & $673[566,808]$ & $675[560,790]$ & $-15[-28,-1]$ & $390[324,440]$ & $374[300,483]$ & $-19[-38,-9]$ & $74[65,84]$ & $71[62,79]$ & $15[13,18]$ \\
\hline \multirow[t]{2}{*}{$\mathrm{O}$} & A & $357[311,393]$ & $353[285,402]$ & $-15[-20,-12]$ & $90[73,104]$ & $84[70,106]$ & $-3[-4,-1]$ & $35[32,38]$ & $35[32,37]$ & $7[6,8]$ \\
\hline & $\mathrm{B}$ & $408[363,472]$ & $387[346,440]$ & $-12[-16,-8]$ & $112[99,132]$ & $116[94,140]$ & $-3[-6,-2]$ & $31[29,33]$ & $31[28,34]$ & $7[6,8]$ \\
\hline \multirow[t]{2}{*}{$\mathrm{U}$} & A & $268[237,328]$ & $252[182,334]$ & $-13[-26,-7]$ & $46[34,59]$ & $44[26,66]$ & $-1[-4,0]$ & $24[21,26]$ & $23[20,26]$ & $5[3,6]$ \\
\hline & $\mathrm{B}$ & $290[235,482]$ & $245[146,382]$ & $-20[-36,-1]$ & $52[38,76]$ & $60[28,98]$ & $-1[-3,2]$ & $17[14,24]$ & $17[13,25]$ & $3[3,5]$ \\
\hline \multirow[t]{2}{*}{ W } & $\mathrm{A}$ & $169[125,196]$ & $77[40,135]$ & $-15[-19,-6]$ & $38[25,56]$ & $29[2,156]$ & $-4[-7,-1]$ & $33[21,41]$ & $32[21,41]$ & $6[4,8]$ \\
\hline & $\mathrm{B}$ & $240[157,330]$ & $69[20,287]$ & $-13[-26,-3]$ & $74[33,87]$ & $60[44,209]$ & $0[-5,4]$ & $31[25,46]$ & $30[22,46]$ & $6[6,9]$ \\
\hline
\end{tabular}

Notes. The values in brackets correspond to the $95 \%$ confidence interval for the median.

${ }^{a}$ Standard deviation of the measurements in the time sequence for each CME. 

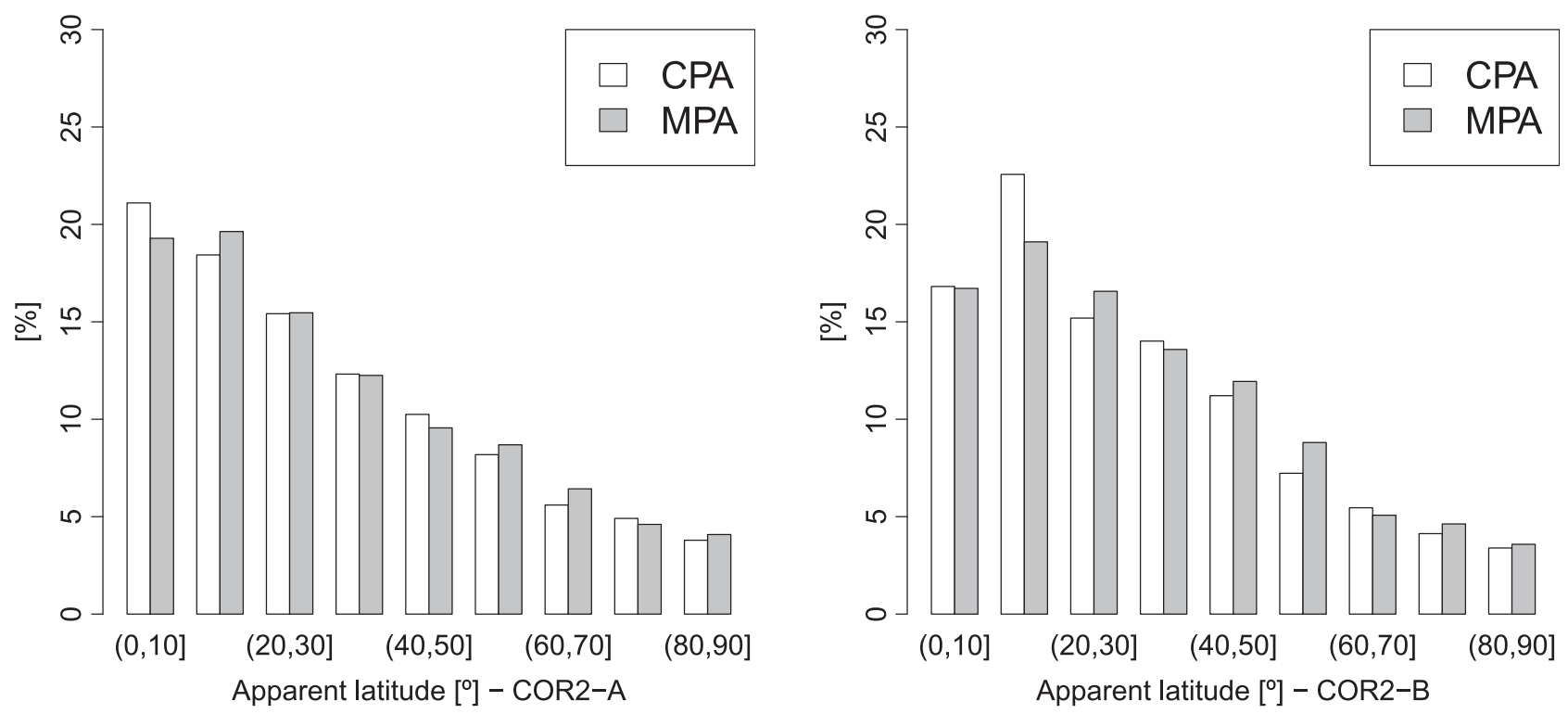

Figure 4. Distribution of CPA and MPA from STA measurements (left) and STB measurements (right).

Overall, the kinematic and angular width distributions derived with the help of CORSET for each CME morphology support our expectations for morphology and the underpinning physical interpretation. That is, L-CMEs are wider than F-CMEs, consistent with the projection of the flux-rope axis into and along the POS, respectively. J-CMEs are narrow, consistent with a jet definition. L-CMEs are faster on average since they are associated mostly with halo or partial halo events, whose halo morphology implies (generally) the presence of a shock (Vourlidas et al. 2013; Kwon et al. 2015). U- and W-type events have the lowest radial and expansion speeds, consistent with their non-flux-rope origins, etc. Therefore, the main conclusion from this section is that the CORSET algorithm results in measurements consistent with previous studies and is therefore a reliable means to measure CME properties across time and event morphology. This gives us confidence to proceed to the main and most novel aspect of this paper, namely the use of simultaneous measurements to evaluate the reliability of single-viewpoint measurements of CMEs.

\subsection{So, How Reliable are Single-viewpoint Measurements after All?}

After establishing that (1) our CME sample is representative of the overall COR2 MVC catalog and (2) CORSET provides consistent measurements between the two COR2 telescopes, we can proceed to assess the reliability of single-viewpoint measurements. We do this by comparing the COR2-A and COR2-B measurements against each other. We compare the radial and expansion speeds, and the angular widths. The radial speeds are computed from height-time measurements at the CPA. We consider only the linear fits for the radial and expansion speeds. The angular widths used here are the median values for each event.

\subsubsection{The Effect of Different Viewing Geometries}

We first investigate projection effects, by binning the measurements according to the POS angular separation between the two spacecraft. For simplicity, we use only three bins to describe the following special viewing geometries: $0^{\circ}-30^{\circ}$ (similar line of sight), $30^{\circ}-60^{\circ}$ (intermediate), and $60^{\circ}-90^{\circ}$ (quadrature). Note that events in the first bin include the configurations of both near alignment and opposition of the spacecraft.

The relations between the STA and STB quantities in each bin are shown as scatter plots in Figure 5. The corresponding least-squares linear fits to the data points are depicted with solid lines. Since we cannot assume either STA or STB measurements as "ground truth," it seems appropriate to estimate the slope by the bisector method described in Isobe et al. (1990). This fit corresponds to the line that bisects the smaller of the two angles between the ordinary least-squares lines: $\operatorname{OLS}(Y \mid X)$ and OLS $(X \mid Y)$. The gray bands show the $95 \%$ confidence intervals for the slope obtained using a bootstrapping technique. The number of points $N$ for each subgroup, the Pearson correlation coefficient $R$, and the slope $b$ are indicated in the inset labels in the plot. The dashed lines mark the slope, $b=1$, corresponding to equal quantities from both viewpoints.

The radial speeds from STA and STB (Figure 5, top panel) agree well with each other with a relatively high degree of correlation $(R>0.8)$, even for large viewpoint separations-a result that should give confidence to the single-viewpoint measurements of the past. The slopes are very close to one for the $[0,30]$ and $[60,90]$ bins of separation angle. For the speeds in the $30^{\circ}-60^{\circ}$ bin, there seems to be a slight bias toward higher speeds in $S T B$ although the value $b=1$ is still within the $95 \%$ confidence interval.

The expansion speeds between COR2-A and -B (Figure 5, middle panel) are within $4 \%$ for all separation angles. The correlation coefficients for the expansion speed are also high $(0.8<R<0.88)$.

The angular widths (Figure 5, lower panel), on the other hand, show much larger differences between the two spacecraft than the speeds. The COR2-A widths differ by at least $15 \%$ from the COR2-B widths for separation angles of $0^{\circ}-60^{\circ}$. There is considerably more scatter than in the comparisons of speeds, with correlation coefficients between 0.66 and 0.83 , with the lowest value in the $30^{\circ}-60^{\circ}$ bin. These results 
reinforce the widely held understanding that the observed CME widths are driven primarily by projection effects.

To visualize whether there is a trend in the differences between the measurements with increasing separation angle between the spacecraft, we plot, in Figure 6 , the ratio of the minimum value to the maximum value for each pair of measurements (regardless of spacecraft) versus the same POS separation bins defined in Figure 5. The boxplots summarize the main characteristics of the samples for each bin. The horizontal line within each box marks the median value for the sample. The lower and upper limits of the box correspond to the first and third quartiles. The extent of the whiskers is 1.5 times the interquartile range (IQR) and points beyond these limits are considered to be outliers (represented by full circles). The mean value of the full sample for each quantity is represented by the horizontal dashed line. We want to determine whether the data in each bin of separation angle differ from the full sample, so we compare the mean values in each bin against the mean of the full sample. Since none of the subsamples follows a normal distribution, we perform a Wilcoxon rank sum test to check whether the differences between the means of the individual boxplots and the overall sample mean are statistically significant. The statistically significant results $(p<0.05)$ are marked with asterisks (“***," $=p<0.001, \quad$ "***, $=p<0.01, \quad$ "**" $=p<0.05$, no asterisk $=p \geqslant 0.05$ ).

The plots in Figure 6 (left panel) suggest that the projection effects on speeds may not be as severe as one would expect, at least in a statistical sense. We find insufficient evidence for a viewpoint effect in the mean speed, since none of our three subsamples of radial speed passed the Wilcoxon rank sum test. Even the IQRs for the three separation bins are very similar. For individual events, projection effects may lead to speeds over/underestimated by as much as a factor of 3, i.e., the boxplot whiskers for the $30^{\circ}-60^{\circ}$ bin, or even a factor of 5 when we look at the outliers.

No clear trend is observed for the ratio between the expansion speeds from both spacecraft, with median values of $0.7<$ ratio $<0.8$ for all bins (Figure 6 , middle panel). As in the case of radial speeds, there is insufficient evidence at the 0.05 level to conclude that the means for each bin differ significantly from the mean of the full sample (Wilcoxon test, $p>0.05$ in all cases). The spread varies from bin to bin, with the largest IQR $(0.54-0.9)$ for the $30^{\circ}-60^{\circ}$ bin and the smallest $(0.61-0.84)$ for the $60^{\circ}-90^{\circ} \mathrm{bin}$.

The widths are very similar for POS separation angles $<30^{\circ}$, when the spacecraft were aligned or at opposition, with a median value for the ratio of 0.86 (Figure 6, right panel). We note a decreasing trend in the angular width ratios with increasing POS distance. The comparison of the means in each group and the mean of the whole sample yields statistically significant differences only for POS angle $<30^{\circ}$. This lends statistical support to the well-known fact that the CME apparent width is strongly dependent on the viewing angle (i.e., the angular distance from the direction of propagation to the spacecraft line of sight). In other words, the same CME may have a very different widths when viewed from different perspectives.

\subsubsection{Error Estimates}

The extended angular coverage allows us to quantify the uncertainty in the CME measurements. We define the relative error between the measurements from the two spacecraft $\epsilon_{i}$ as

$$
\epsilon_{i}=\frac{y_{i}-x_{i}}{\frac{1}{2}\left(x_{i}+y_{i}\right)}
$$

where $x_{i}$ and $y_{i}$ represent a given measurement by $S T A$ or $S T B$ respectively. Since we do not know what the true value is, we normalize the residual by the mean of both measurements. We then use $\epsilon_{i}$ to estimate the residual standard error, RSE:

$$
\mathrm{RSE}=\sqrt{\frac{\sum_{i} \epsilon_{i}^{2}}{N}} .
$$

The RSE values for each quantity in each bin of separation angle are listed in Table 3.

To assess the uncertainty in CME measurements from a single viewpoint we analyze the statistics in the first bin $\left(0^{\circ}-30^{\circ}\right)$, when the viewpoints (and CME appearance) should be almost identical. The first uncertainty arises from the selection of the CME feature used for the measurement of height-time and subsequently speed. Here, the speeds are determined by a semi-automated algorithm and the variation between the two COR2s could be due to small changes in the CME contrast relative to the background. A similar argument can be made for human observers with biases arising from the selection of a different background (i.e., running difference versus base difference, the contrast used, or preconceptions of what constitutes a CME structure). Therefore, we interpret this $39 \%$ level as the baseline accuracy of speeds derived from single-viewpoint measurements made by different observers or with different instruments but made from similar viewpoints. As expected, the differences increase with larger separation angles between the spacecraft (being 18\% larger for quadrature) when the projection effects become important. Our conclusion is that the radial speed derived from a single viewpoint is, on average, reliable to about $40 \%-50 \%$ and is largely independent of viewing geometry. Expansion speeds, on the other hand, are scarce in the literature and it is unclear to what extent projection effects may affect them. We find slightly larger errors than for the radial speeds, from $45 \%$ to $57 \%$, with the largest values in the $30^{\circ}-60^{\circ}$ bin. Errors in the angular widths increase with POS separation angle, in agreement with Figure 6. For events occurring when the spacecraft POSs were less than $30^{\circ}$ apart, the errors are the smallest, about $32 \%$.

\subsubsection{The Effect of Event Morphology}

We repeat the analysis of the previous section but this time we divide the CMEs according to their morphological type. We recall that the classification was performed independently on the images from each spacecraft. We focus on three groups (see discussion around Table 1): F-type for both spacecraft $(\mathrm{F}-\mathrm{F})$, events lacking obvious flux-rope characteristics $(\mathrm{O}-\mathrm{O})$, and For L-type for only one of the spacecraft (F-Other). The first group includes the L-L CMEs, since in our interpretation they are flux-rope CMEs seen edge-on. In the second group, most of the CMEs are classified as O-type, but we include the J-, U-, and $\mathrm{W}$-types, which are considerably fewer in number.

With this, we intend to investigate the effect, if any, of the morphology on the accuracy of the measurements. In Figure 7 we show the scatter plots for the comparisons of simultaneous measurements, with the slope of the linear fit, the correlation coefficient, the standard error, and the number of points indicated in each panel. 
$0 \quad 500 \quad 1000 \quad 15002000$
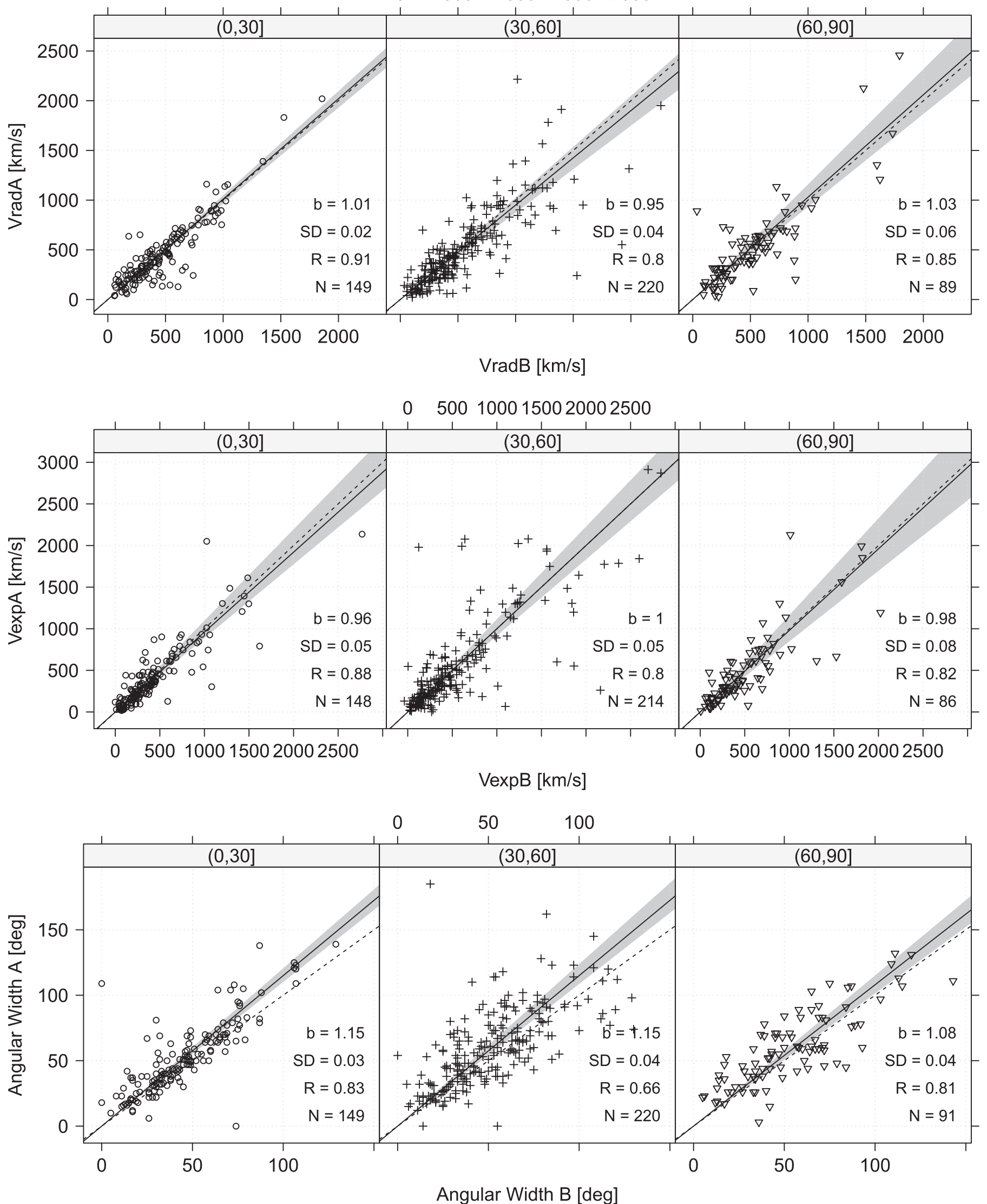

Figure 5. COR2-A vs. COR2-B radial speeds (top row), expansion speeds (middle), and angular widths (bottom row) for the same events as measured by CORSET. Ranges of POS angle are shown on the top of each panel. The solid line shows the regression line through the origin. The dashed line indicates the linear fit with slope $=1$. The correlation coefficient $R$, the slope $b$, the standard error for the slope, and the number of data points $N$ are indicated. The gray bands mark the $95 \%$ confidence interval for the slope. 

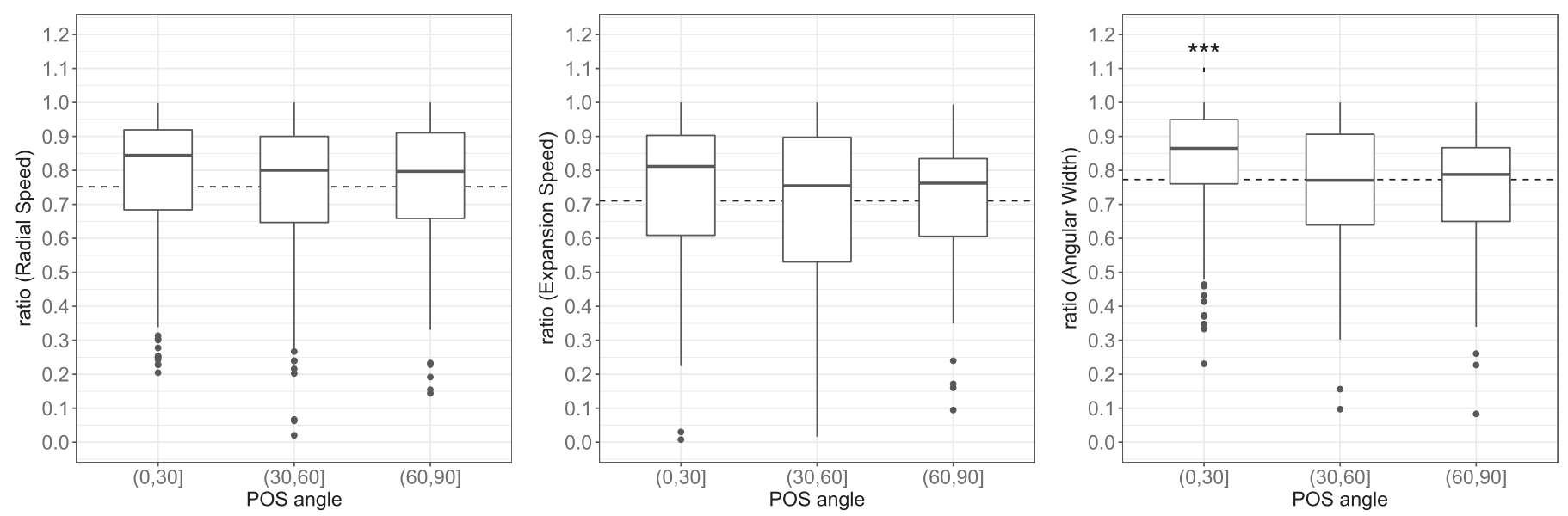

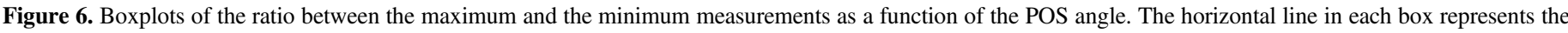

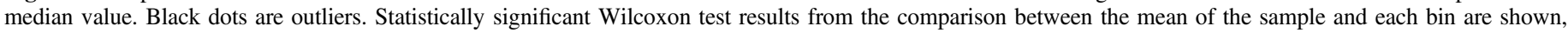
$p<0.001\left(^{* * *}\right)$

We find no obvious trend for any of the quantities when the measurements are grouped according to CME morphology. The radial speeds are well correlated, with correlation coefficients $R>0.83$ for all the types (Figure 7, upper panel). Expansion speeds show lower correlation coefficients $(0.76<R<0.89$, Figure 7 , middle panel). On the other hand, the angular widths show poorer correlations (Figure 7, lower panel). On average, speeds derived from both telescopes agree within $6 \%$, regardless of the morphological type. Similar to the results obtained by grouping the data according to the separation angle, angular widths from $S T B$ are smaller on average than those from STA.

Overall, the measurements are very similar for both spacecraft, irrespective of the morphological type. These results seem to indicate that there is no direct effect of the morphology when comparing simultaneous multi-viewpoint measurements, with the differences arising mainly from the viewing geometry.

\subsection{Triangulated CME Speeds and Directions}

The scatter plots in Figure 8 compare the deprojected speeds estimated from the triangulation technique (described in Section 2.2) against the projected speeds from COR2-A (top row) and COR2-B (bottom row), grouped by POS separation angle. They reveal some interesting trends. The correlation coefficients are low $(R<0.4)$ for POS separation angles below $30^{\circ}$. The multi-viewpoint benefits disappear when the two telescopes have similar lines of sight through the CME; the CME appears very similar in both telescopes. This is the case for separations below $30^{\circ}$, where the Thomson scattering efficiency becomes insensitive to angular changes (see, e.g., Figure 3 in Vourlidas et al. 2010). The average difference between projected and deprojected values in this bin of POS separation angle is about $43 \%$ for STA and $47 \%$ for $S T B$. The optimal range of separation angles for triangulations is $60^{\circ}-90^{\circ}$, as we show with the following exercise.

We examine the errors introduced by the deprojection technique by computing the condition number of the linear system represented in Equations (2)-(7) in Section 2.2 as a function of the POS separation angle. The condition number represents the amplification factor between variations in the
Table 3

RSE Determined from the Comparison of Multi-viewpoint Measurements

\begin{tabular}{lccc}
\hline \hline & \multicolumn{3}{c}{ POS Separation Angle } \\
\cline { 2 - 4 } & {$\left[0^{\circ}, 30^{\circ}\right]$} & {$\left[30^{\circ}, 60^{\circ}\right]$} & {$\left[60^{\circ}, 90^{\circ}\right]$} \\
\hline Radial speed & $39 \%$ & $45 \%$ & $47 \%$ \\
Expansion speed & $45 \%$ & $57 \%$ & $46 \%$ \\
Angular width & $32 \%$ & $41 \%$ & $45 \%$ \\
\hline
\end{tabular}

data (measured speeds) and the solution of the linear system (deprojected speeds). Figure 9 shows the ratio between the deprojected speeds and the measured speeds as a function of time and therefore the POS separation angle. The blue line shows the variation of the condition number. High condition numbers suggest that the solutions are unstable with respect to small changes in the data, and hence the triangulated speeds and directions are more uncertain. The lowest condition numbers occur when the spacecraft are in quadrature (at $45^{\circ}$ and $135^{\circ}$ from Earth) and rapidly increase when the spacecraft are aligned or in opposition (the two spacecraft have similar views of the $\mathrm{CME}$ and thus the linear system becomes singular).

Therefore, the triangulation analysis shows that: (1) two viewpoints with POS separated by $60^{\circ}-90^{\circ}$ provide the most reliable inputs for speed triangulation, (2) triangulations for POS separations below $30^{\circ}$ should be avoided, and (3) the projected CME speeds are, on average, underestimated by $\sim 22 \%-28 \%$; namely, $\quad v_{\text {deproj }}^{A}=(1.22 \pm 0.04) V_{\text {rad }}^{A} \quad$ and $\quad v_{\text {deproj }}^{B}=(1.28 \pm$ 0.04) $V_{\mathrm{rad}}^{B}$.

In addition to the deprojected speeds, the triangulation procedure gives the event direction and consequently the location of the source region, if we assume radial propagation (see Figure 10). We find that almost two thirds of the events $(63 \%)$ originate within the equatorial belt $\left( \pm 30^{\circ}\right.$ latitude), and another third $(\sim 33 \%)$ originates at latitudes of $30^{\circ}-60^{\circ}$. Only $4 \%$ of the CMEs appear to come from higher latitudes, which may reflect the relatively short period of solar maximum observations in our sample (2012-2014 September). As expected, CME latitudes increase with time as streamers appear at higher latitudes toward the maximum phase of the cycle (Figure 10, left). The figure indicates the north-south 

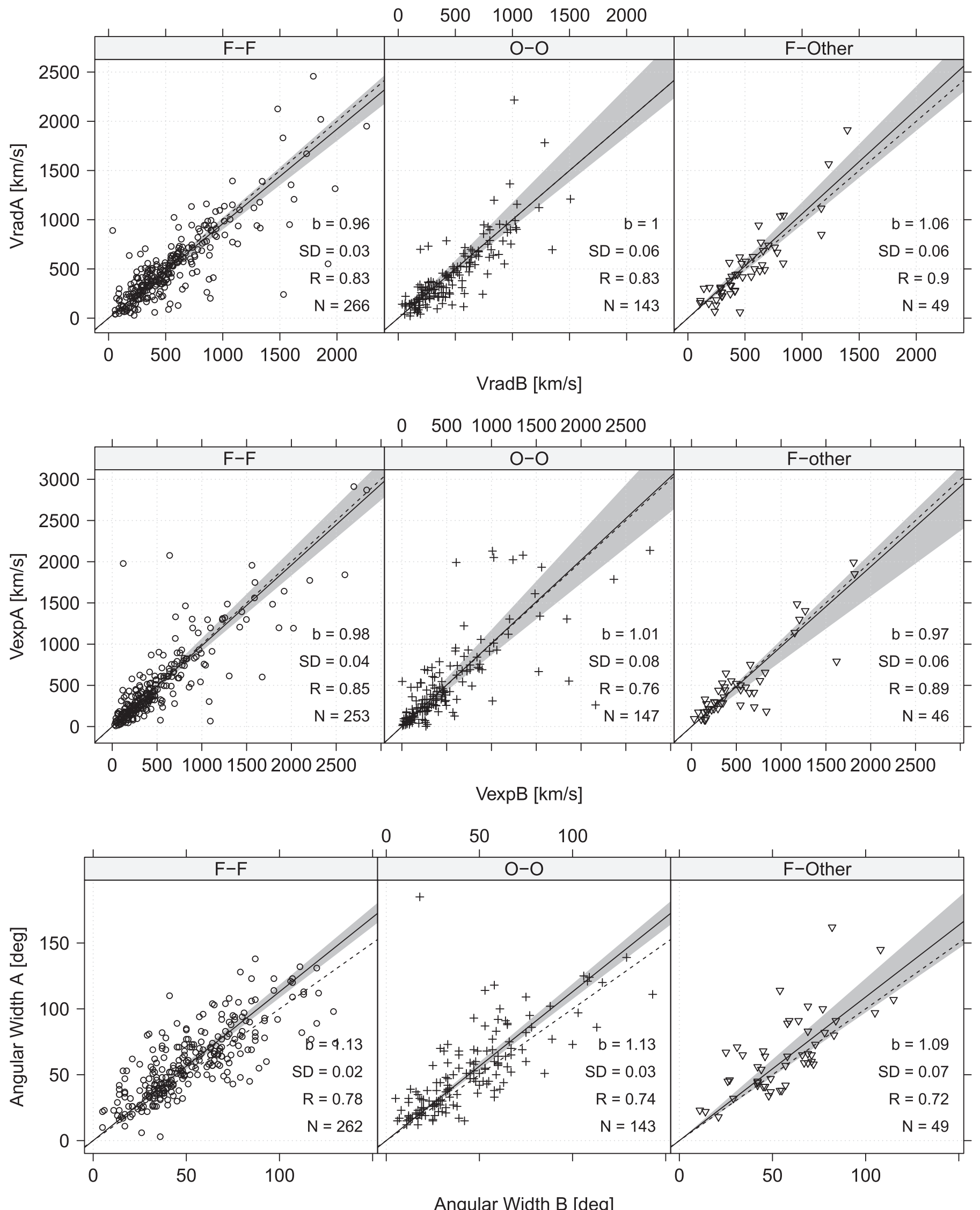

Figure 7. Scatter plot of the COR2-A vs. COR2-B radial speeds (top row), expansion speeds (middle), and angular widths (bottom row) for the same events as measured by the CORSET algorithm divided into groups according to the morphological classification. The dashed line indicates the line with slope 1 . The solid line corresponds to the linear fit with the slope shown in each panel. The correlation coefficient $R$, the slope $b$, the standard error for the slope, and the number of data points $N$ are indicated. The gray bands mark the $95 \%$ confidence interval for the slope. 

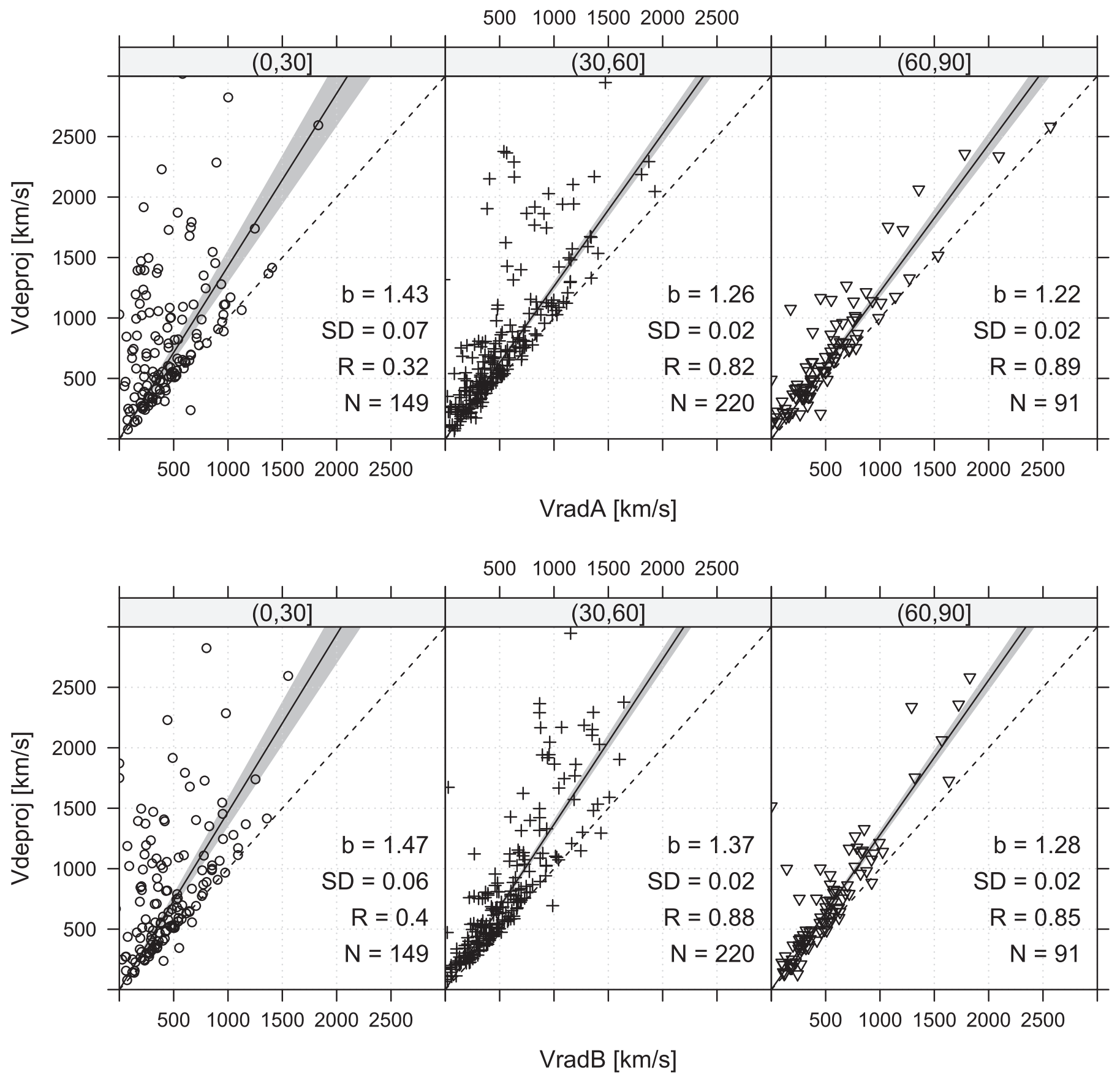

Figure 8. Scatter plots of the deprojected speeds vs. the COR2-A (top row) and COR2-B (bottom row) projected values as measured by the CORSET algorithm, divided into groups according to the POS separation angle. The dashed line corresponds to slope $=1$. The solid line represents the best linear fit with the slope $b$. The correlation coefficient $R$, the slope $b$, the standard error for the slope, and the number of data points $N$ are indicated. The gray bands mark the $95 \%$ confidence interval for the slope.

asymmetry in activity and sunspot numbers seen in the current cycle, with most activity occurring in the north first (2012) and later in the south (2014).

In the right panel of Figure 10, we plot the distribution of Carrington longitudes of events with time. On the side, we show the frequency per longitude bin. There is little variation in the frequencies from bin to bin. We find that $\sim 51 \%$ of the CMEs in our sample originated from the Earth-facing hemisphere with $\sim 20 \%$ of them (i.e., 93 events) launched from near the central meridian $\left(-30^{\circ}<\lambda<30^{\circ}\right)$.

\section{Lessons Learned from Applying a Supervised Computer Vision Algorithm to CME Tracking}

The overall performance of the supervised computer vision algorithm CORSET as well as the direct comparison with measurements from other catalogs have already been addressed and briefly discussed in previous works (see, e.g., Goussies et al. 2010; Braga et al. 2013, 2017). However, the number of events analyzed in these works falls short of meaningful statistics to allow us to draw a general picture of the caveats and benefits of such a technique. 


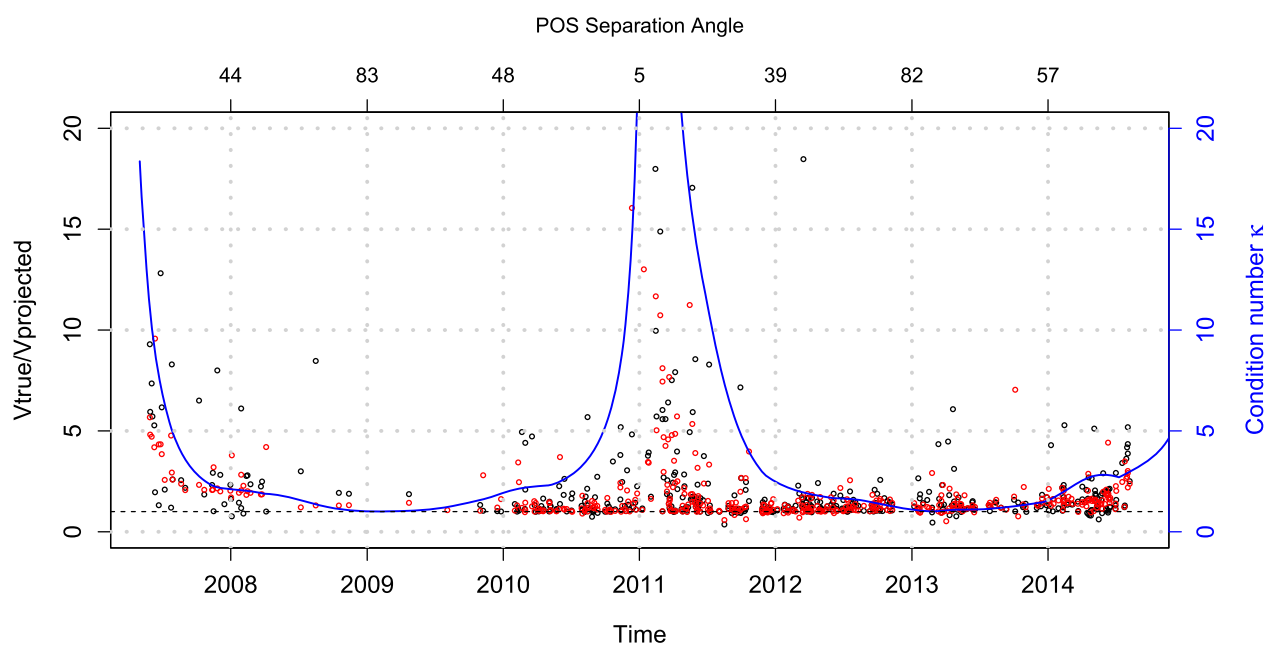

Figure 9. Variation of the condition number of the equation system $\kappa$ (blue curve) with time (and therefore the POS separation angle). Black and red points correspond to the ratio between the deprojected speeds and the projected values from STA and STB respectively.

For this work, we have used the manually cataloged CME events recorded by either of the COR2 coronagraph on board the twin spacecraft of the STEREO mission between 2007 May and 2014 September (Vourlidas et al. 2017), and applied the CORSET algorithm to all events, irrespective of size, width, brightness, speed, etc. Therefore, we have acquired a statistically meaningful data set on which to draw conclusions on the advantages and shortcomings of the technique.

The CORSET detections do not discriminate by intensity, size, or speed but rely solely on the texture of the feature as compared to a given background texture. As is the case with any other segmentation technique based on a probability test, our results show that the segmentation begins to fail when the predictor becomes ambiguous, i.e., when the texture of the feature starts resembling that of the background. We encountered this situation in events that lacked a clear, well-defined boundary. For instance:

1. slow F-CMEs traveling away from the POS. In this case the segmentation results in a concave structure ( $\mathrm{Y}$-shape);

2. J-CMEs or jets, which have sharp lateral boundaries but look like continuous flows in the radial direction. In this case, the segmentation works until the event reaches about $10 R_{\odot}$, when the signal-to-noise ratio begins to drop noticeably;

3. wave-like CMEs, which do not maintain their textural properties as they travel through the FOV. In these cases, the front is well defined early in the event but its contrast (which is one of many textural features conveyed in the GLCM) decreases rapidly with height. Also, these events frequently lack clear lateral boundaries.

In brief, a key parameter, for a segmentation technique based on texture, is the signal-to-noise ratio of the scene, which we will simply refer to here as "roughness." As discussed above, the roughness of the scene depends on the type of event, but it also depends on the image processing performed on the data. We have tried several low- and high-pass filters on the input images and found that the segmentation failed when either too low or too high a frequency signal was removed. This can be intuitively understood by noting that the roughness of the feature of interest and that of the background start to resemble each other despite the difference in intensity.
A crucial step in our texture-based CME tracking technique is the choice of the base image. Many segmentations failed due to a poor choice of background image, because (1) it was too far in time from the first event image; (2) it included a partly overlapping event; (3) it included co-spatial streamers or large comets; (4) it had energetic particles hitting the detector; (5) it contained many saturated pixels with signal bleed. In all these cases, the texture of the feature of interest is altered and hence the predictor no longer serves its purpose. As inferred from the results presented in Section 3.1, a proper selection of the baseimage was feasible in at least 33\% of cases (both instruments combined).

A further complication arises when trying to cross-track the events from two simultaneous viewpoints. As pointed out in Paper I, it is not always possible to simultaneously identify and moreover to segment and track the same CME in both COR2 instruments. The reasons abound: (1) CMEs exhibiting a variety of orientations with respect to the POS have therefore very different morphology and/or textural properties (strongly dependent upon the spacecraft separation); (2) the presence of overlapping events, etc.

In summary, the use of our supervised methodology shows that blind acceptance of the results provided by any automated procedure to track CME events of any kind is not advisable. Supervision is strongly required, and even with supervision the success rate will depend upon the type of event, the phase of the solar cycle, overlapping features/events in the line of sight, etc.

\section{Summary and Conclusions}

We have presented an analysis of the angular widths and kinematic properties of CME events obtained from two vantage points. The properties were derived by applying a supervised image segmentation algorithm, CORSET, to simultaneous observations from the two COR2 telescopes on the STEREO mission from 2007 May to 2014 September. We selected only CMEs that were successfully tracked in both COR2s. The sample of 460 events offers a unique opportunity to test the accuracy and constraints of single-viewpoint properties that underlie the bulk of CME research to date. Here, we analyzed the radial and expansion speeds and angular width of the events both as single-viewpoint quantities and in 3D (via a triangulation 

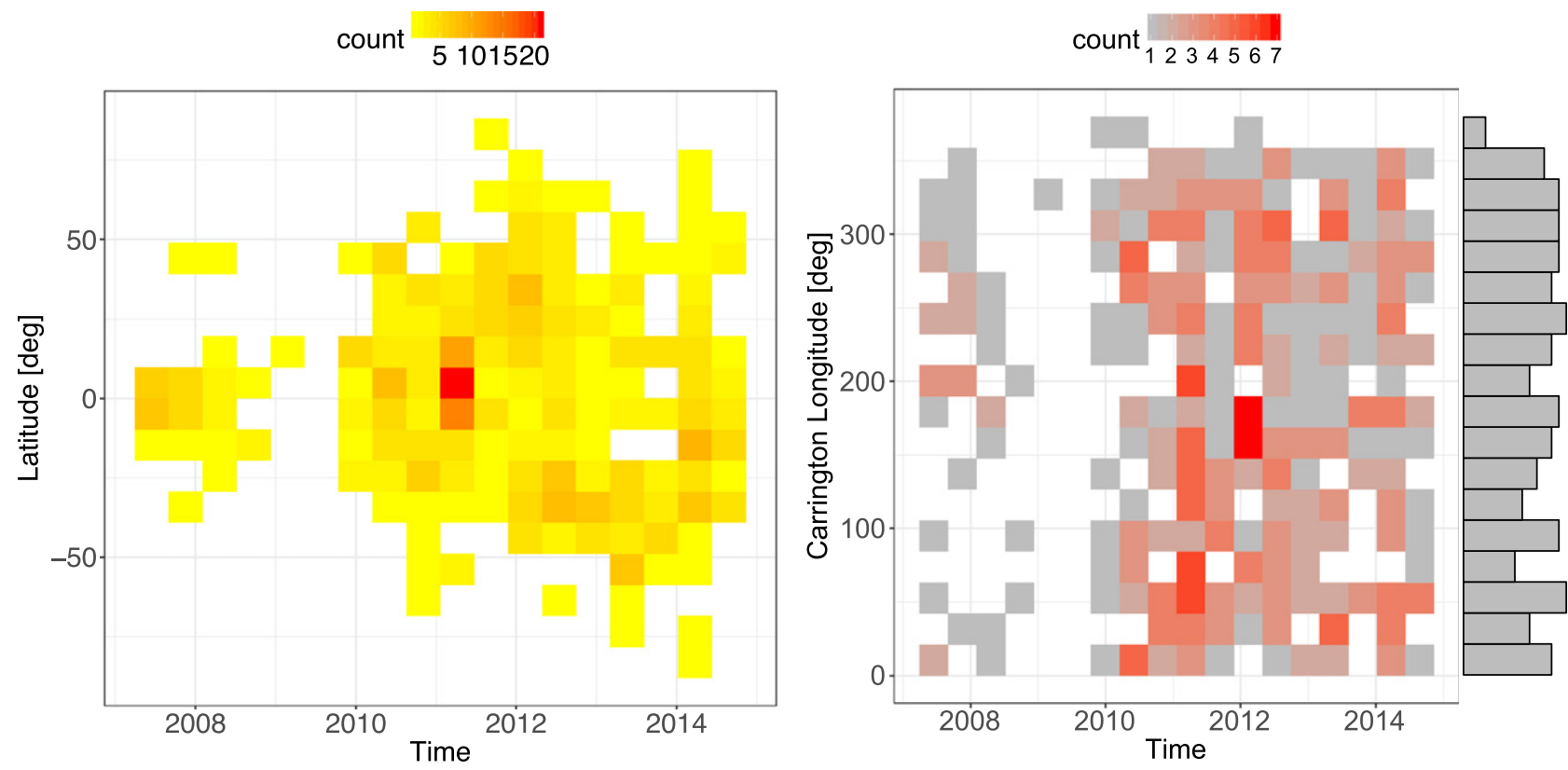

Figure 10. CME source region based on the triangulation results. Left: event latitude vs. time. Right: Carrington longitude vs. time. Each bin corresponds to $20^{\circ}$ in the $y$ direction and approximately 6 months on the $x$ axis. Along the right axis, we plot the event histogram per longitude bin.

technique). In addition, we examined the dependence of these properties on the morphology of the events and attempted to quantify the differences between the measurements from the two instruments with varying spacecraft separation.

From the analysis of the properties derived from singleviewpoint observations, we find that:

1. The widths show some dependence on the CME morphology. They are least constrained for loop-type CMEs and CMEs with ambiguous morphology in one of the telescopes $(\mathrm{F}-\mathrm{O}$ or $\mathrm{L}-\mathrm{O})$. The variation in the L-CMEs can be understood as the effect of better shock detection in COR2-A since these CMEs tend to be large and impulsive.

From the comparison of simultaneous measurements from two viewpoints, we find that:

1. The radial speeds between COR2-A and COR2-B are well correlated $(R=0.8-0.9)$. The best correlation occurs when the spacecraft are closely aligned or in opposition because they have the same view through the CME. Even at quadrature, the two speeds are, on average, within $20 \%$ of each other. This is an encouraging finding for past (and future) single-viewpoint kinematic studies. Speeds of individual events, however, can differ by up to a factor of 5 .

2. Taking advantage of their simultaneity, the scatter between COR2-A and -B radial speeds at small spacecraft separations provides a measure of the uncertainty in the measurement of the speed of the same event by different observers or with different instruments. We find the intrinsic uncertainty for the radial speeds to be $39 \%$.

3. Expansion speeds are rarely reported in the literature but the CORSET algorithm enables that measurement for all of the events in our sample. Expansion speeds from different viewpoints show the same trends as the radial speeds (i.e., well correlated between COR2-A and -B with $R>0.8$ ). They are on average within $57 \%$ of each other for all separation angles (see Table 3).

4. The widths are not as well correlated as the speeds between the two telescopes $(R=0.66-0.83)$. This is expected since the widths are more susceptible to projection effects than the other parameters.

5. The COR2-A widths are systematically larger than the COR2-B widths. We attribute this to the higher signal-tonoise ratio of COR2-A, which improves the detection of the fainter outer boundaries of CMEs and possibly shocks.

6. Overall, and rather reassuringly, we find no indication that the accuracy of speed or width measurements depends strongly on the event morphology. We do find, however, that the morphology plays a role in the success of the segmentation algorithm.

From the application of a rather simple triangulation technique to the projected speeds (Sections 2.2 and 3.4), we deduce the following:

1. Triangulations from dual viewpoints can lead to reliable 3D speeds and directions, if the viewpoint separations are wide enough to offer different views of the events, i.e., POS separation angles between $30^{\circ}$ and $90^{\circ}$. The best results occur for separations of $60^{\circ}-90^{\circ}$ (Figure 9). Therefore, CME imaging from the Lagrangian viewpoints $\mathrm{L}_{5}$ and $\mathrm{L}_{4}$ (up to $90^{\circ}$ ) could provide reliable measurements of Earth-directed events.

2. A $10 \%$ uncertainty in the projected speeds (singleviewpoint measurements) translates into errors of the order of $50 \%$ or more for periods when the POS separation angle is smaller than $20^{\circ}$. Therefore, speed triangulations at such small angles should be avoided.

3. The projected CME speeds are, on average, underestimated by $\sim 22 \%-28 \%$ (Figure 8 ).

Regarding the performance of CORSET, we emphasize that the algorithm was originally developed for and tested against 
large and fast events, and it was designed to give overall morphological characteristics of CMEs. This is the first time we test its capabilities on other types of CME events (e.g., faint, with diffuse boundaries, etc.). We find that CMEs exhibiting unclear boundaries (e.g., with faint or fuzzy borders such as jets, or wave-like CMEs) are difficult to distinguish from the background. In these cases, the segmentation becomes challenging (as it does with all algorithms currently available). In particular, we conclude that height-time measurements for jet-like CMEs are not as reliable as those for larger CMEs. However, their widths seem to be properly measured. The CORSET success rate for STA $(28 \%)$ was about $10 \%$ higher than for STB. The overall success rate of CORSET is $33 \%$ (considering both spacecraft combined over the total number of unique events in the MVC).

In this work, we have reported on the first compilation of kinematic measurements of hundreds of CMEs (460 events) from dual viewpoints, over the full range of separation angles of the spacecraft $\left(0^{\circ}-180^{\circ}\right)$ and phases of the solar cycle (minimum to peak of solar activity of cycle 24). The analysis permitted us to assess, in detail, the reliability of singleviewpoint kinematic measurements of CMEs from a variety of perspectives, namely in terms of the measurement technique, event morphology, and viewpoint. Our findings, summarized earlier in this section, should hopefully be a useful guide in the use of automated algorithms for extraction of the physical parameters of CMEs, in the interpretation of single-viewpoint observations (likely to be the norm after the end of the STEREO mission), and in the formulation of empirical corrections for space weather purposes.

The SECCHI data are courtesy of STEREO and the SECCHI consortium. The SECCHI data are produced by an international consortium of the NRL, LMSAL, and NASA GSFC (USA), RAL and University of Birmingham (UK), MPS (Germany), CSL (Belgium), IOTA and IAS (France). L.A.B. was supported by FAPESP Projects 2014/23716-4 and 2013/03085-7 and NASA grant NNX17AC47G. She also thanks JHUAPL for their hospitality during her FAPESP fellowship. A.V. is supported by
NASA grant NNX16AH70G and NNX17AC47G. G.S. acknowledges the support from the NASA STEREO/SECCHI (NNG17PP27I) program. A.D.L. thanks CNPq (grant 304209/ 2014-7). The CME list and APL Solar site are supported by NASA NNX16AH70G and APL internal funds.

\section{ORCID iDs}

Laura A. Balmaceda (10 https://orcid.org/0000-00031162-5498

Angelos Vourlidas (ib https://orcid.org/0000-0002-8164-5948 Guillermo Stenborg (iD https://orcid.org/0000-00018480-947X

Alisson Dal Lago (iD https://orcid.org/0000-0002-4361-6492

\section{References}

Braga, C. R., Dal Lago, A., Echer, E., Stenborg, G., \& Rodrigues Souza de Mendonça, R. 2017, ApJ, 842, 134

Braga, C. R., Dal Lago, A., \& Stenborg, G. 2013, AdSpR, 51, 1949

Brueckner, G. E., Howard, R. A., Koomen, M. J., et al. 1995, SoPh, 162, 357

Burkepile, J. T., Hundhausen, A. J., Stanger, A. L., St. Cyr, O. C., \& Seiden, J. A. 2004, JGRA, 109, A03103

Cremades, H., \& Bothmer, V. 2004, A\&A, 422, 307

Frazin, R. A., Vsquez, A. M., Thompson, W. T., et al. 2012, SoPh, 280, 273 Gopalswamy, N., Yashiro, S., Akiyama, S., et al. 2008, AnGeo, 26, 3033

Goussies, N., Stenborg, G., Vourlidas, A., \& Howard, R. 2010, SoPh, 262, 481

Haralick, R. M., Shanmugam, K., \& Dinstein, I. 1973, ITSMC, 3, 610

Howard, R. A., Moses, J. D., Vourlidas, A., et al. 2008, SSRv, 136, 67

Isobe, T., Feigelson, E. D., Akritas, M. G., \& Babu, G. J. 1990, ApJ, 364, 104

Kaiser, M. L., Kucera, T. A., Davila, J. M., et al. 2008, SSRv, 136, 5

Kwon, R.-Y., Zhang, J., \& Vourlidas, A. 2015, ApJL, 799, L29

Lee, H., Moon, Y.-J., Na, H., Jang, S., \& Lee, J.-O. 2015, JGRA, 120, 10237

Mierla, M., Davila, J., Thompson, W., et al. 2008, SoPh, 252, 385

Robbrecht, E., \& Berghmans, D. 2004, A\&A, 425, 1097

Shen, C., Wang, Y., Pan, Z., et al. 2013, JGRA, 118, 6858

Temmer, M., Preiss, S., \& Veronig, A. M. 2009, SoPh, 256, 183

Vourlidas, A., Balmaceda, L. A., Stenborg, G., \& Dal Lago, A. 2017, ApJ, 838,141

Vourlidas, A., Howard, R. A., Esfandiari, E., et al. 2010, ApJ, 722, 1522

Vourlidas, A., Lynch, B. J., Howard, R. A., \& Li, Y. 2013, SoPh, 284, 179

Wood, B. E., Wu, C.-C., Lepping, R. P., et al. 2017, ApJS, 229, 29

Yashiro, S., Gopalswamy, N., Michalek, G., et al. 2004, JGRA, 109, A07105

Zhang, J., Richardson, I. G., Webb, D. F., et al. 2007, JGRA, 112, 10102 